Louisiana State University

LSU Digital Commons

Faculty Publications

Department of Chemistry

$10-14-2017$

\title{
Ultrafast and nonlinear spectroscopy of brilliant green-based nanoGUMBOS with enhanced near-infrared emission
}

Tony E. Karam

Louisiana State University

Noureen Siraj

Louisiana State University

Zhenyu Zhang

Louisiana State University

Abdulrahman F. Ezzir

Louisiana State University

Isiah M. Warner

Louisiana State University

See next page for additional authors

Follow this and additional works at: https://digitalcommons.Isu.edu/chemistry_pubs

\section{Recommended Citation}

Karam, T., Siraj, N., Zhang, Z., Ezzir, A., Warner, I., \& Haber, L. (2017). Ultrafast and nonlinear spectroscopy of brilliant green-based nanoGUMBOS with enhanced near-infrared emission. Journal of Chemical Physics, 147 (14) https://doi.org/10.1063/1.4994712

This Article is brought to you for free and open access by the Department of Chemistry at LSU Digital Commons. It has been accepted for inclusion in Faculty Publications by an authorized administrator of LSU Digital Commons.

For more information, please contact ir@lsu.edu. 


\section{Authors}

Tony E. Karam, Noureen Siraj, Zhenyu Zhang, Abdulrahman F. Ezzir, Isiah M. Warner, and Louis H. Haber 


\section{Ultrafast and nonlinear spectroscopy of brilliant green-based nanoGUMBOS with enhanced near-infrared emission}

Tony E. Karam, Noureen Siraj, Zhenyu Zhang, Abdulrahman F. Ezzir, Isiah M. Warner, and Louis H. Haber

Citation: The Journal of Chemical Physics 147, 144701 (2017);

View online: https://doi.org/10.1063/1.4994712

View Table of Contents: http://aip.scitation.org/toc/jcp/147/14

Published by the American Institute of Physics

\section{Articles you may be interested in}

2D IR spectroscopy of high-pressure phases of ice

The Journal of Chemical Physics 147, 144501 (2017); 10.1063/1.4993952

Distance-including rigorous upper bounds and tight estimates for two-electron integrals over long- and shortrange operators

The Journal of Chemical Physics 147, 144101 (2017); 10.1063/1.4994190

Coherent laser-millimeter-wave interactions en route to coherent population transfer

The Journal of Chemical Physics 147, 144201 (2017); 10.1063/1.4997624

Multivalent cation induced attraction of anionic polymers by like-charged pores

The Journal of Chemical Physics 147, 144901 (2017); 10.1063/1.4994018

An intrinsic representation of atomic structure: From clusters to periodic systems

The Journal of Chemical Physics 147, 144106 (2017); 10.1063/1.4997292

A transition-state based rotational sudden (TSRS) approximation for polyatomic reactive scattering

The Journal of Chemical Physics 147, 144104 (2017); 10.1063/1.5003226

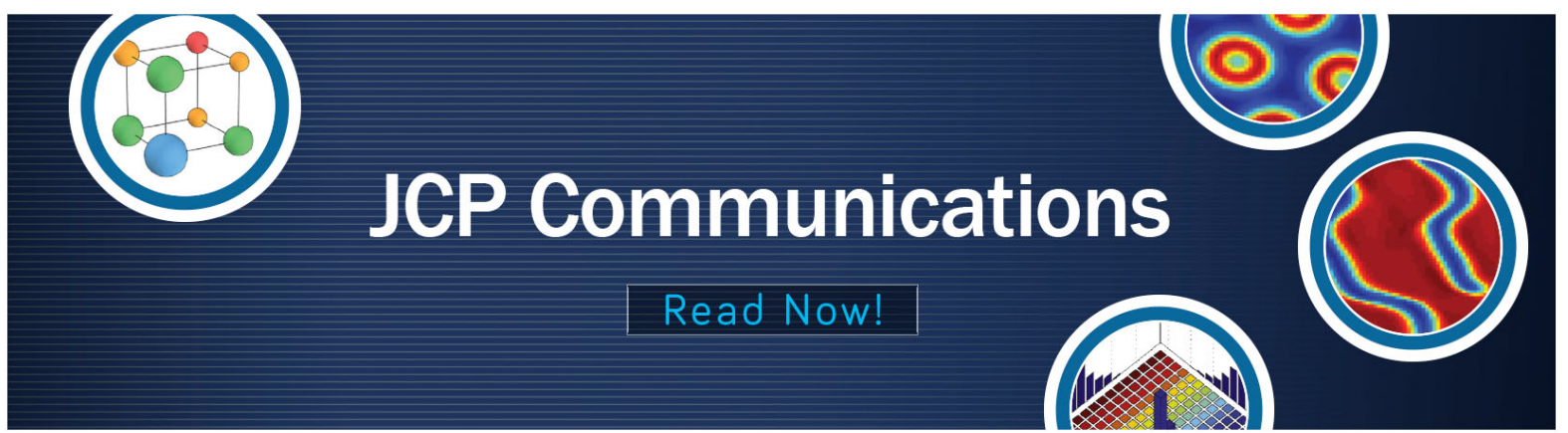




\title{
Ultrafast and nonlinear spectroscopy of brilliant green-based nanoGUMBOS with enhanced near-infrared emission
}

\author{
Tony E. Karam, ${ }^{1, a)}$ Noureen Siraj, ${ }^{1,2}$ Zhenyu Zhang, ${ }^{1}$ Abdulrahman F. Ezzir, ${ }^{1}$ \\ Isiah M. Warner, ${ }^{1}$ and Louis H. Haber ${ }^{1, b)}$ \\ ${ }^{1}$ Department of Chemistry, Louisiana State University, Baton Rouge, Louisiana 70803, USA \\ ${ }^{2}$ Department of Chemistry, University of Arkansas at Little Rock, Little Rock, Arkansas 72204, USA
}

(Received 6 July 2017; accepted 22 September 2017; published online 9 October 2017)

\begin{abstract}
The synthesis, characterization, ultrafast dynamics, and nonlinear spectroscopy of $30 \mathrm{~nm}$ nanospheres of brilliant green-bis(pentafluoroethylsulfonyl)imide ([BG] [BETI]) in water are reported. These thermally stable nanoparticles are derived from a group of uniform materials based on organic salts (nanoGUMBOS) that exhibit enhanced near-infrared emission compared with the molecular dye in water. The examination of ultrafast transient absorption spectroscopy results reveals that the overall excited-state relaxation lifetimes of $[\mathrm{BG}][\mathrm{BETI}]$ nanoGUMBOS are longer than the brilliant green molecular dye in water due to steric hindrance of the torsional degrees of freedom of the phenyl rings around the central carbon. Furthermore, the second harmonic generation signal of [BG][BETI] nanoGUMBOS is enhanced by approximately 7 times and 23 times as compared with colloidal gold nanoparticles of the same size and the brilliant green molecular dye in water, respectively. A very clear third harmonic generation signal is observed from the [BG][BETI] nanoGUMBOS but not from either the molecular dye or the gold nanoparticles. Overall, these results show that [BG][BETI] nanoGUMBOS exhibit altered ultrafast and nonlinear spectroscopy that is beneficial for various applications including nonlinear imaging probes, biomedical imaging, and molecular sensing. Published by AIP Publishing. https://doi.org/10.1063/1.4994712
\end{abstract}

\section{INTRODUCTION}

Investigations into a group of uniform materials based on organic salts (GUMBOS), which are composed of frozen ionic liquids and related organic salts with melting points ranging from 25 to $250^{\circ} \mathrm{C}$, provide potential applications in sensing, labeling, and optoelectronics due to exceptional tunability of their chemical and optical properties. ${ }^{1}$ Colloidal nanoparticles prepared from GUMBOS are termed nanoGUMBOS and have been recently studied as candidates for photovoltaics, ${ }^{2,3}$ biomedical imaging, ${ }^{4}$ and molecular sensing. ${ }^{5-7}$ Previously, we have reported on ultrafast excited-state dynamics of colloidal crystalline ruthenium bipyridine-bis(pentafluoroethylsulfonyl) imide $\left(\left[\mathrm{Ru}(\mathrm{bipy})_{3}\right]-[\mathrm{BETI}]_{2}\right)$ that show spectral shifts and sizedependent relaxation dynamics. ${ }^{3}$ These $\left[\mathrm{Ru}(\text { bipy })_{3}\right]-[\mathrm{BETI}]_{2}$ nanoGUMBOS exhibit long-lived phonon oscillations with size-dependent frequencies that increase as the nanoparticle size increases due to very low coupling between electronic and phonon degrees of freedom and a strong hydrophobic interaction with the aqueous solvent. ${ }^{3}$ However, nanoGUMBOS composed from different dye molecules can have dramatically different mechanisms for ultrafast excited-state relaxation dynamics. Additionally, nonlinear

\footnotetext{
a)Present address: Division of Chemistry, California Institute of Technology, Pasadena, California 91125, USA.

b)Author to whom correspondence should be addressed: 1haber@1su.edu. Telephone: (225) 578-7965.
}

spectroscopy of nanoGUMBOS has not been reported previously and provides fundamental insights into molecular-based nanomaterials that are important for developing biologically relevant nonlinear optical imaging applications.

Triphenylmethane dyes such as malachite green (MG) and brilliant green (BG) have been widely studied for biological molecular labeling. ${ }^{89}$ Low fluorescence yields of MG and BG in solution are observed due to ultrafast nonradiative relaxation dynamics of the first excited singlet state as a result of strong coupling between electronic states and torsional degrees of freedom of the phenyl ring around the central carbon. ${ }^{10-12}$ These "dark" molecules are capable of generating enhanced fluorescence signals when specifically bound to proteins or nucleic acids due to the molecular confinement of the phenyl ring. ${ }^{13-16}$ Several strategies have been employed to enhance near-infrared (NIR) emission of triphenylmethane dyes corresponding to the optical window in biological tissues for potential applications in non-invasive bio-imaging and bio-sensing. ${ }^{17,18}$ In this regard, the use of nanoGUMBOS chemistry can offer tunable photophysical properties due to the presence of bulky counterions which affect free rotation of phenyl rings around the central carbon and spectral red-shifting due to dye aggregation.

Second harmonic generation (SHG) and third harmonic generation (THG) are nonlinear optical processes where two or three photons of frequency $\omega$ add together coherently to generate a photon of frequency $2 \omega$ or $3 \omega$, respectively. ${ }^{19-21}$ Nonlinear optical harmonic generation and SHG, in 
particular, are of increasing interest for various applications including photonics, ${ }^{22-24}$ materials chemistry, ${ }^{25}$ biosensing, ${ }^{26}$ and biomedical imaging. ${ }^{27}$ Molecules and nanomaterials with enhanced SHG signals are useful probes for second harmonic generation microscopy and imaging. ${ }^{28,29}$ SHG microscopy is uniquely suited for biological imaging due to the narrow and stable nonlinear signal generated at the laser focus, the surface sensitivity of the spectroscopy, and the upconversion of NIR incident light to visible signals. ${ }^{30,31}$ Several triphenylmethane dyes, such as MG and BG, are useful as nonlinear optical probes for molecular adsorption and transport studies in colloidal nanoparticle samples. ${ }^{32-35}$ Recently, it was reported that triphenylmethane-based nanoparticles are excellent candidates for single particle tracking and bioimaging due to their enhanced fluorescence and second harmonic signal. ${ }^{36-38}$ Molecular-based nanomaterials such as nanoGUMBOS offer a new route for designing nonlinear optical probes by combining the inherent molecular nonlinear susceptibilities with the ability to control the resulting nanoparticle size as well as molecular interactions and structural ordering.

In this manuscript, we report the synthesis, characterization, excited-state dynamics, and nonlinear optical spectroscopy of brilliant green-bis(pentafluoroethylsulfonyl)imide ([BG][BETI]) nanoGUMBOS. These thermally stable colloidal nanomaterials exhibit enhanced NIR fluorescence as compared with the molecular dye in water. The examination of ultrafast spectroscopy data shows that the excited-state lifetimes of brilliant green in the nanoGUMBOS are significantly longer due to molecular hindrance. The longer lifetimes together with spectral red-shifting due to dye aggregation lead to the enhanced NIR fluorescence of the nanoGUMBOS for potential applications in fluorescence labeling. Additionally, the nonlinear signals of the nanoGUMBOS samples are investigated. The SHG signal of the [BG][BETI] nanoGUMBOS is enhanced approximately 23 -fold as compared with the brilliant green molecular dye in water due to an increase in the effective second-order susceptibility tensor $\chi^{(2)}$ of the nanoGUMBOS compared with the free dye. A THG signal is clearly observed from the colloidal nanoGUMBOS samples, while no THG signal is observed from the free dye in water at the same concentration. These results demonstrate that $[\mathrm{BG}][\mathrm{BETI}]$ nanoGUMBOS are promising candidates for biological labeling, sensing, and nonlinear optical imaging applications.

\section{EXPERIMENTAL SECTION}

\section{Synthesis and characterization}

Brilliant green (BG.HSO $\left.{ }_{4}\right)$ and lithium bis(pentafluoroethylsulfonyl)imide (LiBETI) were purchased from Sigma Aldrich and Iolitech, respectively, and used as received. Ethanol (EtOH) was purchased from OmniSolv. Triply deionized ultrapure distilled water $(18.2 \mathrm{M} \Omega \mathrm{cm})$ is used for all ion exchange reactions, sample preparations, and spectroscopic measurements. The molecular structures of BG and BETI are shown in Fig. 1.

A metathesis approach is first used to synthesize brilliant green GUMBOS. In this regard, 0.13 mol of BG.HSO 4
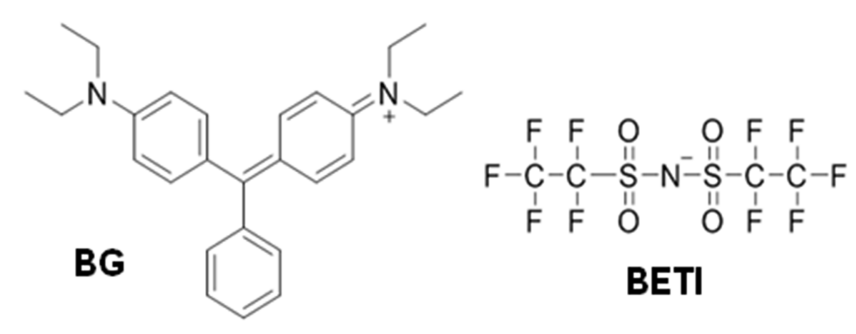

FIG. 1. Molecular structures of BG and BETI in the nanoGUMBOS.

and $0.14 \mathrm{~mol}$ of LiBETI are dissolved in separate and equal volumes of water. An anion exchange reaction is performed between BG.HSO $_{4}$ and LiBETI by mixing these two solutions and stirring the mixture for $24 \mathrm{~h}$, followed by centrifugation at $3800 \mathrm{rpm}$ to separate the [BG][BETI] blue-green precipitate. In order to separate the lithium bisulphate byproduct that is soluble in water, the $[\mathrm{BG}][\mathrm{BETI}]$ precipitate is washed several times with fresh deionized distilled water. The $[\mathrm{BG}][\mathrm{BETI}]$ product is then freeze-dried overnight to remove trace water, providing an overall product yield of approximately $99 \%$. The [BG][BETI] nanoGUMBOS sample is prepared in water using a reprecipitation method. Briefly, a small aliquot $(50 \mu \mathrm{l})$ of $1 \mathrm{mM}$ [BG][BETI] ethanolic solution is dissolved in a vial containing $5 \mathrm{ml}$ of deionized distilled ultrapure water in an ice bath, followed by 5 min of probe sonication using $25 \%$ power. A probe sonicator (model VCX 133 750, Sonics and Materials, Inc.) with a $6.5 \mathrm{~mm}$ tapered microtip is employed for nanoparticle preparations.

The $[\mathrm{BG}][\mathrm{BETI}]$ nanoGUMBOS sample is characterized using transmission electron microscopy (TEM), extinction spectroscopy, and fluorescence spectroscopy. A survey of more than one hundred TEM images is used to determine the average nanoGUMBOS diameter to be $30 \pm 5 \mathrm{~nm}$. A representative TEM image of the nanoGUMBOS is shown in Fig. 2. Additional TEM images as well as a histogram of the size distribution are shown in the supplementary material. A $0.4 \mathrm{~cm}$ path length quartz cuvette is used for absorbance measurements against an identical cell filled with water as the blank solvent. A $0.4 \mathrm{~cm}$ path length quartz cuvette is used for fluorescence measurements using $420 \mathrm{~nm}$ excitation at right angle geometry. Additional details and characterizations of [BG][BETI] including thermogravimetric analysis, differential scanning

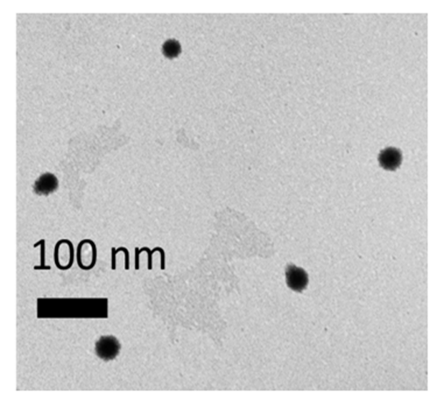

FIG. 2. TEM image of the $[\mathrm{BG}][\mathrm{BETI}]$ nanoGUMBOS with an average diameter of $30 \pm 5 \mathrm{~nm}$. 
calorimetry, NMR measurements, and mass spectrometry are described in the supplementary material.

\section{Nonlinear spectroscopy}

The nonlinear spectroscopy setup is composed of an ultrafast laser system, an optical setup, and a high-sensitivity charge-coupled device (CCD) spectroscopy detector. ${ }^{39,40}$ Briefly, a titanium:sapphire oscillator laser produces $75 \mathrm{fs}$ pulses centered at $800 \mathrm{~nm}$ with a repetition rate of $80 \mathrm{MHz}$ and an average power of $2.7 \mathrm{~W}$, which is attenuated to $900 \mathrm{~mW}$ for the nonlinear spectroscopic measurements. A $20 \mathrm{~mm}$ focal length lens is used to focus the laser beam into a $1 \mathrm{~cm}$ quartz cuvette containing the colloidal nanoGUMBOS sample. An optical filter is placed in front of the cuvette to remove any residual SHG or THG light prior to the sample. Another filter is placed after the sample to remove fundamental light while transmitting SHG and THG signals, which are collected in the forward direction and refocused to a monochromator connected to a high-sensitivity spectroscopy CCD detector.

\section{Transient absorption spectroscopy}

The transient absorption spectroscopy setup has been previously described. ${ }^{3,41,42}$ Briefly, an amplified titanium:sapphire laser system produces $0.7 \mathrm{~mJ}$, $75 \mathrm{fs}$ pulses centered at $800 \mathrm{~nm}$ with a repetition rate of $10 \mathrm{kHz}$. A beam splitter is used to separate the pump and probe pulses, and a retroreflector on a computer-controlled translation stage is used to control the pump-probe temporal delay. The pump beam is passed through an optical parametric amplifier (OPA) to generate the $400 \mathrm{~nm}$ laser pulses. The probe beam is passed through a nonlinear crystal, $\beta$-barium borate (BBO), to generate the second harmonic signal centered at $400 \mathrm{~nm}$ through frequency doubling. The $400 \mathrm{~nm}$ beam and the residual $800 \mathrm{~nm}$ beam are focused into a fused quartz flow cell containing water to generate the femtosecond white light probe pulses that are refocused to a spatial overlap with the pump pulse at the sample, which is contained in a $3 \mathrm{~mm}$ fused quartz flow cell. Spectra are acquired from 400 to $1000 \mathrm{~nm}$ using a fiber optic spectrometer detector. A computercontrolled beam block is used in synchronization with an automated file saving program to generate transient absorption spectra $(\Delta \mathrm{A})$. Several time-resolved spectral scans are acquired for each sample in order to generate reliable statistical analysis.

\section{RESULTS AND DISCUSSION}

Figure 3(a) is a display of the extinction spectra of $10 \mu \mathrm{M}$ brilliant green dye in water and $10 \mu \mathrm{M}$ colloidal [BG][BETI] nanoGUMBOS in water, where the nanoGUMBOS concentration is the concentration of [BG][BETI]. The absorption peaks near $420 \mathrm{~nm}$ and $620 \mathrm{~nm}$ correspond to $S_{0}$ to $S_{2}$ and $S_{0}$ to $S_{1}$ transitions, respectively. ${ }^{10,12,18,43}$ The extinction spectrum of the nanoGUMBOS closely follows the corresponding dye spectrum over the wavelength range of $300 \mathrm{~nm}-550 \mathrm{~nm}$. The higher extinction of the nanoGUMBOS below $300 \mathrm{~nm}$ is due to increased
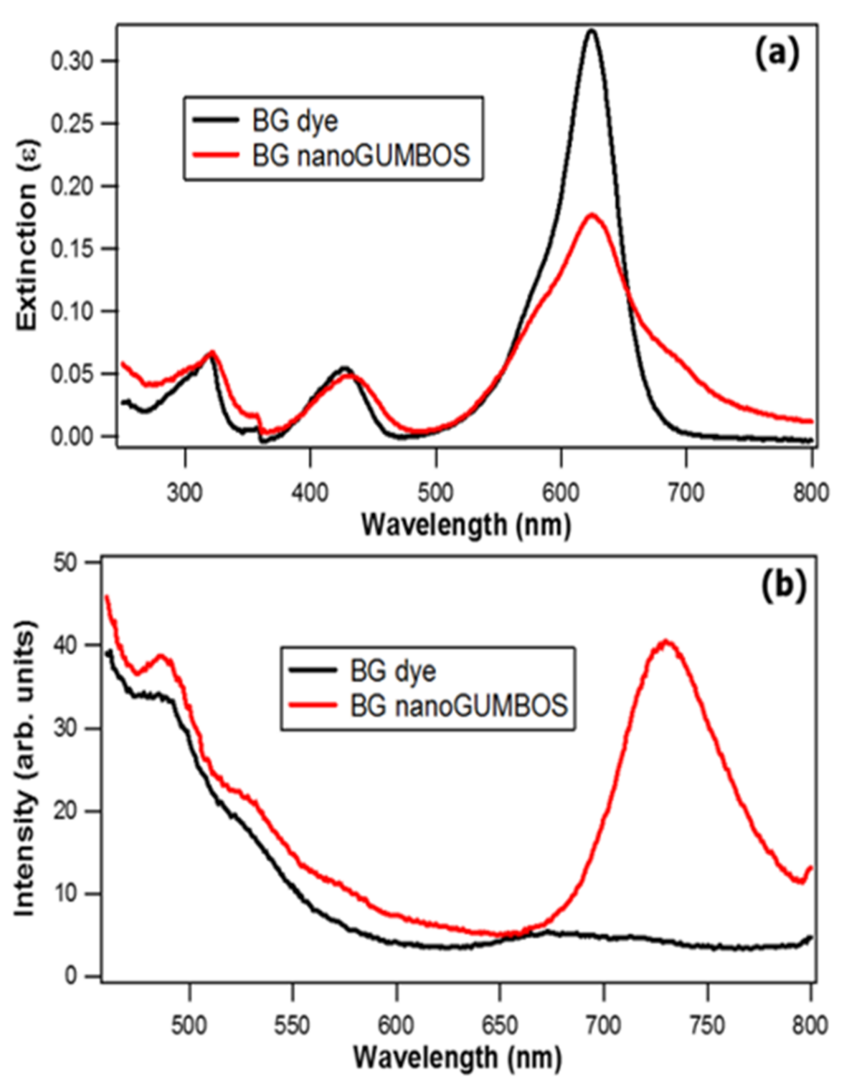

FIG. 3. (a) Extinction spectra and (b) fluorescence spectra of the $10 \mu \mathrm{M}$ brilliant green dye (black line) and $10 \mu \mathrm{M}$ [BG][BETI] nanoGUMBOS (red line) in water.

scattering by these colloidal nanoparticles. ${ }^{18}$ The extinction spectral deviations between the nanoGUMBOS and the BG dye from $550 \mathrm{~nm}$ to $800 \mathrm{~nm}$ are attributed to dye aggregation and different chemical environments in the amorphous nanoGUMBOS nanoparticle. The fluorescence spectra of brilliant green dye and [BG][BETI] nanoGUMBOS using $420 \mathrm{~nm}$ excitation are shown in Fig. 3(b). The fluorescence spectrum of the nanoGUMBOS closely resembles the spectrum of the BG dye in the wavelength region of $450 \mathrm{~nm}-650 \mathrm{~nm}$. However significantly enhanced NIR fluorescence centered around $740 \mathrm{~nm}$ is observed in nanoGUMBOS. The NIR fluorescence peak is approximately 6 times larger in the nanoGUMBOS as compared with the BG dye, which has a much weaker fluorescence peak centered around $670 \mathrm{~nm}$. The fluorescence signal of the BG dye from $500 \mathrm{~nm}$ to $600 \mathrm{~nm}$ is attributed to $S_{2}$ to $S_{0}$ emission, while the fluorescence signal from $620 \mathrm{~nm}$ to $750 \mathrm{~nm}$ is attributed to $S_{1}$ to $S_{0}$ emission. ${ }^{11}$ This observation suggests that the $S_{2}$ to $S_{0}$ emission peak is comparable in the $B G$ dye and [BG][BETI] nanoGUMBOS, whereas significance enhancement in the $S_{1}$ to $S_{0}$ NIR emission peak is observed in the $[\mathrm{BG}][\mathrm{BETI}]$ nanoGUMBOS sample in comparison with the BG parent dye. The observed NIR fluorescence enhancement is attributed to molecular confinement and is consistent with previous studies on triphenylmethane structures specifically bound to proteins or nucleic acids and malachite green based nanoparticles. ${ }^{13-16,18}$ The red-shifting of the fluorescence and absorption bands suggests the formation of J-aggregates in the nanoGUMBOS. 
Ultrafast transient absorption is used to investigate the excited-state dynamics of the [BG][BETI] nanoGUMBOS in order to understand the mechanism of the enhanced NIR fluorescence. For direct comparison, the excited-state dynamics of the BG dye in water is first investigated. Figure 4(a) shows representative transient absorption spectra of the $10 \mu \mathrm{M}$ brilliant green dye in water at different time delays using $400 \mathrm{~nm}$ excitation. The positive band centered at $480 \mathrm{~nm}$ is from excited-state absorption. The negative bands centered at $580 \mathrm{~nm}$ and $650 \mathrm{~nm}$ are attributed to the bleach of the $\mathrm{S}_{0}-\mathrm{S}_{1}$ absorption as well as stimulated emission. ${ }^{10}$ The time-profiles of the brilliant green parent dye measured at 480 and $580 \mathrm{~nm}$ are shown in Fig. 4(b). The decay spectra obtained from global analysis ${ }^{10,42}$ of the transient absorption time-profiles of the brilliant green dye in water are shown in Fig. 4(c), based on fitting the wavelengthdependent dynamics to sums of multiple exponential decays. Three lifetimes $\tau_{1}=0.63 \pm 0.03 \mathrm{ps}, \tau_{2}=0.82 \pm 0.04 \mathrm{ps}$,
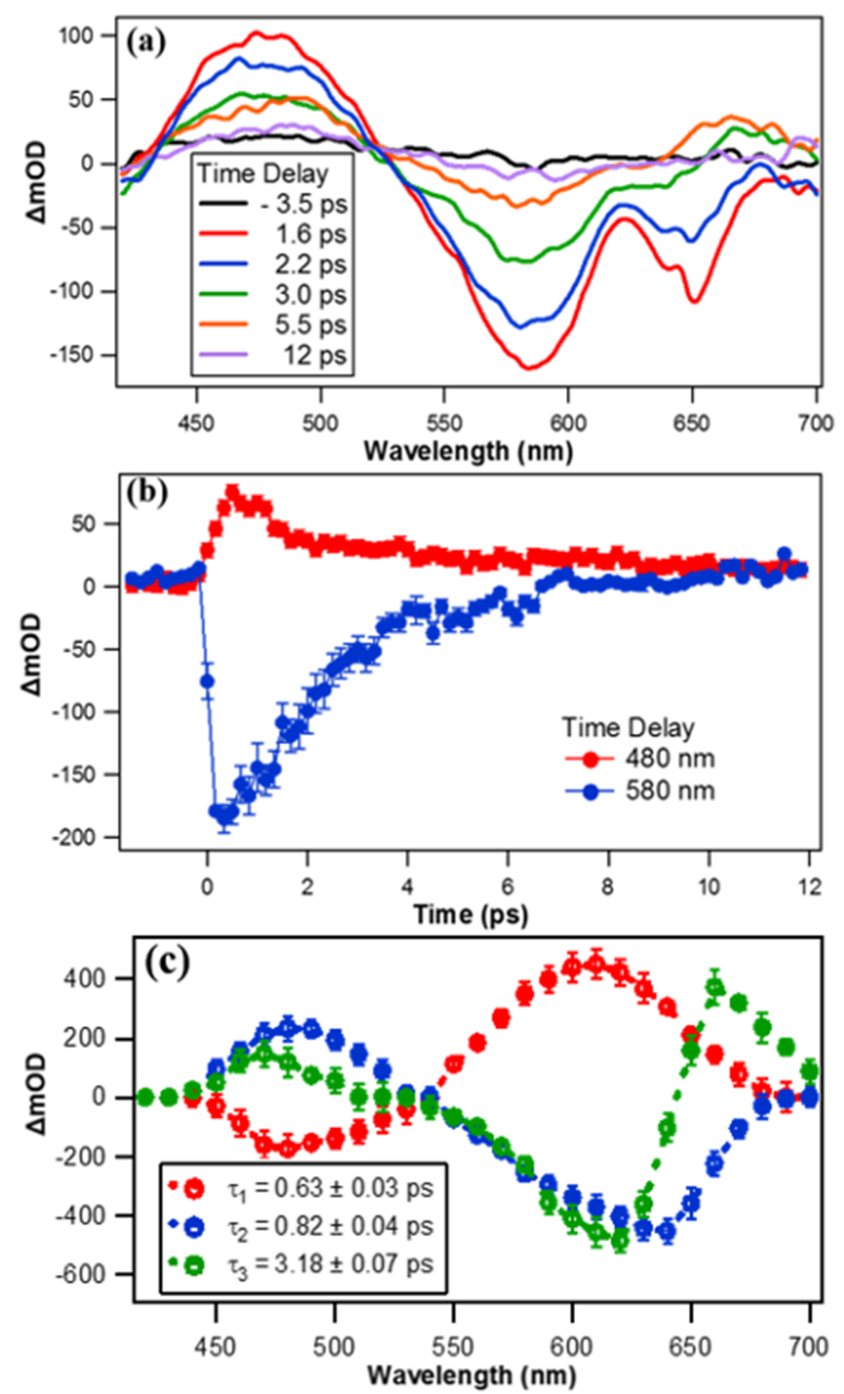

FIG. 4. (a) Representative transient absorption spectra of the $10 \mu \mathrm{M}$ brilliant green dye in water at different time delays using $400 \mathrm{~nm}$ excitation. (b) Representative time-profiles of the brilliant green dye measured at 480 and $580 \mathrm{~nm}$. (c) Decay spectra obtained from global analysis of the transient absorption time-profiles of the brilliant green dye. The amplitude of the decay spectrum at $\tau_{3}$ is multiplied by a factor of 7 for clarity purposes. and $\tau_{3}=3.18 \pm 0.07 \mathrm{ps}$ are needed to accurately describe the ultrafast excited-state dynamics of brilliant green in water. The results of dynamics and corresponding spectra obtained for the brilliant green parent dye are in agreement with previously published measurements, noting the similarities in the excitedstate relaxation dynamics of BG and MG. ${ }^{10-12}$ Previous work on MG showed very similar lifetimes and transient absorption spectra when exciting with $615 \mathrm{~nm}$ and $400 \mathrm{~nm} .{ }^{10}$ Additionally, the same ultrafast relaxation mechanism was observed for $\mathrm{BG}$ and MG in viscosity-dependent solvent mixtures under $615 \mathrm{~nm}$ excitation. ${ }^{12}$ However, detailed transient absorption spectroscopy studies have not been previously conducted on BG using $400 \mathrm{~nm}$ excitation, to the best of our knowledge. The transient absorption decay spectra of BG measured here with $400 \mathrm{~nm}$ excitation are very similar to previous studies using $615 \mathrm{~nm}$ excitation. ${ }^{12}$ The fastest lifetime $\tau_{1}$ is attributed to the ultrafast relaxation of the Frank-Condon $S_{2}$ state to the structurally relaxed $S_{1}$ state through a conical intersection. ${ }^{11,12}$ The second two lifetimes $\tau_{2}$ and $\tau_{3}$ are ascribed to the torsional twist and twist-back, respectively, of phenyl rings around the central carbon in the $S_{1}$ to $S_{0}$ nonradiative relaxation through an $S_{x}$ twisted propeller-like conformer of the excited ground state. ${ }^{10,12}$ The spectra obtained from global analysis have positive and negative peaks near the $S_{0}-S_{1}$ and $\mathrm{S}_{0}-\mathrm{S}_{2}$ absorption transitions, with the spectrum associated with $\tau_{1}$ having approximately opposite magnitudes compared to the spectra associated with $\tau_{2}$ and $\tau_{3}$. This suggests that these excited states and corresponding depleted states have spectra that are dominated by increases and decreases in these optical transitions. These nonradiative pathways are in competition with the simultaneous radiative relaxation from the $\mathrm{S}_{2}$ and $\mathrm{S}_{1}$ excited states to the $\mathrm{S}_{0}$ ground state, ${ }^{11}$ so increases in the lifetimes will lead to corresponding increases in the fluorescence.

Excited-state dynamics of colloidal [BG][BETI] nanoGUMBOS in water are measured and compared with the corresponding dynamics of the brilliant green dye in order to understand the effect of the altered chemical environment. Figure 5(a) shows representative transient absorption spectra of $10 \mu \mathrm{M}$ [BG][BETI] nanoGUMBOS at different time delays using $400 \mathrm{~nm}$ excitation. A positive band centered at $540 \mathrm{~nm}$ is attributed to excited-state absorption, followed by a negative band from ground-state depletion centered at $650 \mathrm{~nm}$. The time profiles of colloidal [BG][BETI] nanoGUMBOS measured at 540 and $650 \mathrm{~nm}$ are shown in Fig. 5(b). The decay spectra obtained from global analysis of the transient absorption time-profiles of the [BG][BETI] nanoGUMBOS are shown in Fig. 5(c). Three lifetimes $\tau_{1}=0.46 \pm 0.03 \mathrm{ps}$, $\tau_{2}=1.12 \pm 0.09 \mathrm{ps}$, and $\tau_{3}=4.31 \pm 0.13 \mathrm{ps}$ are needed to accurately describe the ultrafast excited-state dynamics of the [BG][BETI] nanoGUMBOS. As observed from the transient absorption results, both $\tau_{2}$ and $\tau_{3}$ are significantly longer in the nanoGUMBOS as compared with the BG molecular dye in aqueous solution. These longer lifetimes are attributed to the restriction of the torsional degrees of freedom of the BG phenyl rings around the central carbon atom in the nanoGUMBOS environment. Previous transient absorption spectroscopic studies of the BG molecular dye in water-glycerol mixtures demonstrate that $\tau_{2}$ and $\tau_{3}$ are slower at higher viscosity due 

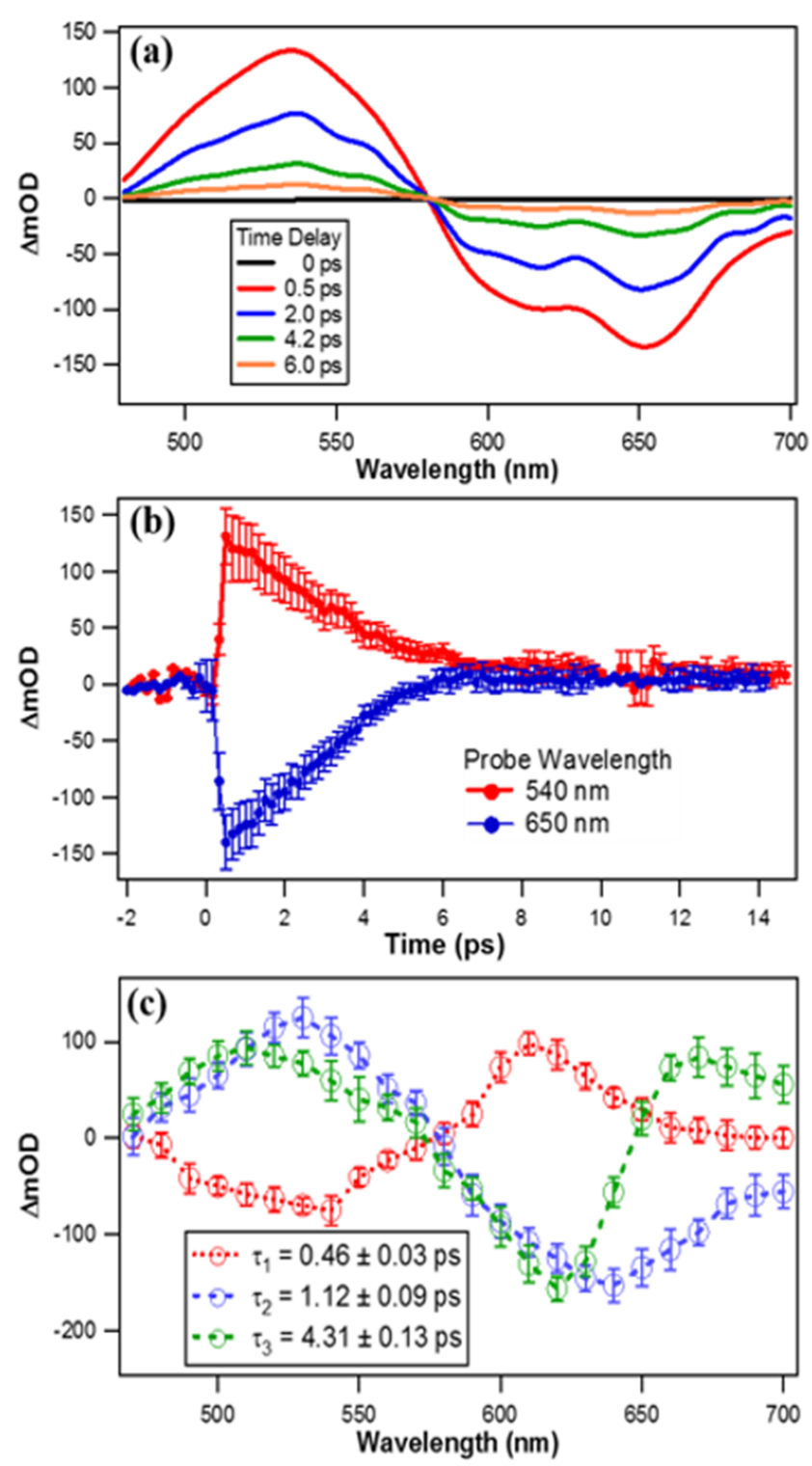

FIG. 5. (a) Representative transient absorption spectra of $10 \mu \mathrm{M}$ [BG][BETI] nanoGUMBOS in water at different time delays using $400 \mathrm{~nm}$ excitation. (b) Representative time-profiles of [BG][BETI] nanoGUMBOS measured at 540 and $650 \mathrm{~nm}$. (c) Decay spectra obtained from global analysis of the transient absorption time-profiles of [BG][BETI] nanoGUMBOS. The amplitude of the decay spectrum at $\tau_{3}$ is multiplied by a factor of 7 for clarity purposes.

to hindrance of the torsional dynamics of the phenyl rings, leading to enhanced fluorescence from the $S_{1}$ state. ${ }^{12} \mathrm{~A}$ similar effect is observed in nanoGUMBOS, where both $\tau_{2}$ and $\tau_{3}$ are slower in the BG nanoparticle than in the BG solution due to torsional restraint of the phenyl rings. However, the changes in lifetime are not due to viscosity of the medium but rather the aggregation and altered chemical environment of the BG dye in nanoGUMBOS. Interestingly, $\tau_{1}$ is slightly faster in nanoGUMBOS, possibly due to changes in the BG structure and corresponding ultrafast relaxation from $\mathrm{S}_{2}$ to $\mathrm{S}_{1}$ in nanoGUMBOS. A red-shift in the transient absorption spectra of the [BG][BETI] nanoGUMBOS is observed as compared with the BG dye, both in the depletion and excitedstate absorption signals as well as the corresponding global analysis decay spectra. These red shifts are attributed to dye aggregation and different chemical environments of the BG molecule in the nanoGUMBOS form in colloidal suspension in water. ${ }^{46}$ Together, these results demonstrate that steric hindrance of the phenyl ring, increased lifetimes of $\tau_{2}$ and $\tau_{3}$, and red-shifting of the $S_{1}$ to $S_{0}$ transition leads to the observation of enhanced NIR fluorescence of the [BG][BETI] nanoGUMBOS as compared with the BG molecular dye.

In a related investigation, nonlinear spectroscopic signals from second harmonic generation and third harmonic generation of the $[\mathrm{BG}][\mathrm{BETI}]$ nanoGUMBOS in water are recorded and compared with corresponding signals acquired from the BG dye in water at the same concentration. These results are further compared with $35 \pm 3 \mathrm{~nm}$ colloidal gold nanospheres at the same gold concentration. Colloidal gold nanoparticle samples have been extensively studied using second harmonic generation measurements, providing a useful benchmark for the SHG results..$^{19,40,47-49}$ More details on the colloidal gold nanoparticle sample are included in supplementary material. Figs. 6(a) and 6(b) show the SHG and THG spectra, respectively, of $10 \mu \mathrm{M}$ brilliant green molecular dye in water, $10 \mu \mathrm{M}$ of $30 \mathrm{~nm}$ [BG][BETI] nanoGUMBOS in water, and $10 \mu \mathrm{M}$ of $35 \mathrm{~nm}$ gold nanospheres in water. The SHG spectra are fit using Gaussian functions and the obtained intensities of the peaks are $1362 \pm 2,202 \pm 4$, and $60 \pm 4$ for [BG][BETI] nanoGUMBOS, gold nanospheres in water, and brilliant green molecular dye in water, respectively. Notably, the SHG signal observed in the [BG][BETI] nanoGUMBOS is $6.7 \pm 0.1$ times larger than the SHG signal from the gold nanospheres and $23 \pm 1$ times larger than the SHG signal from the BG dye in water.
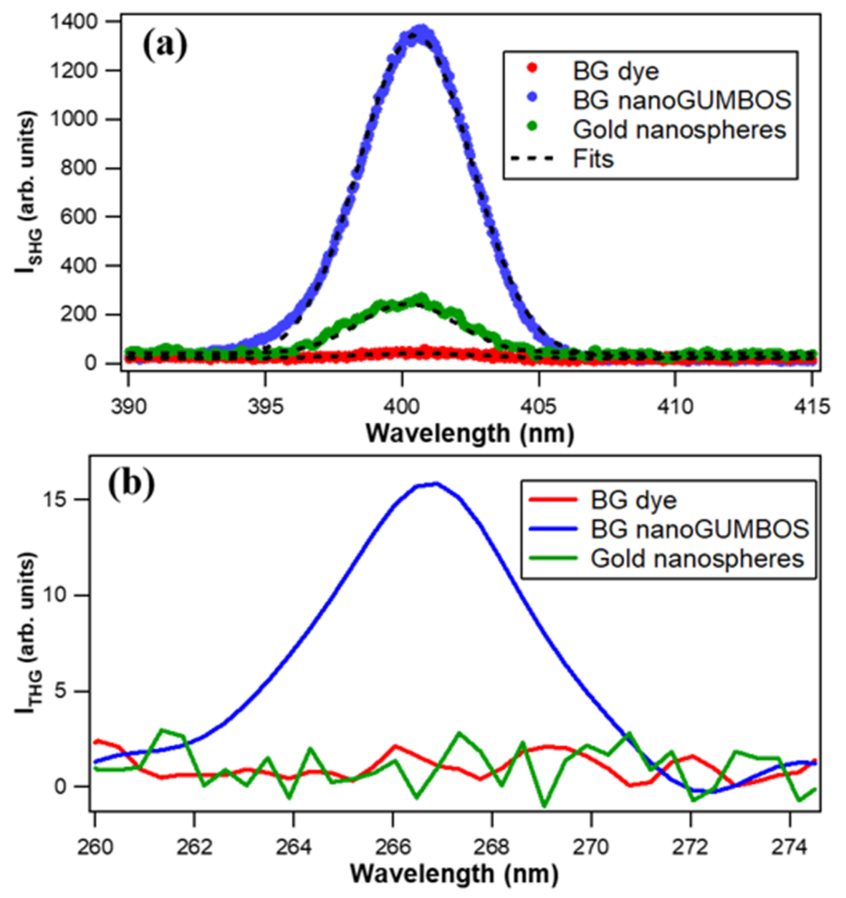

FIG. 6. (a) SHG and (b) THG spectra of (red line) $10 \mu \mathrm{M}$ brilliant green dye in water, (blue line) $10 \mu \mathrm{M}$ of $30 \pm 5 \mathrm{~nm}$ [BG][BETI] nanoGUMBOS in water, and (green line) $10 \mu \mathrm{M}$ of $35 \pm 3 \mathrm{~nm}$ gold nanospheres in water. The SHG signals are fit using Gaussian functions. 
The enhanced SHG signal of the nanoGUMBOS as compared with the molecular dye is caused by a larger effective $\chi^{(2)}{ }^{50,51}$ The BG molecule exhibits a two-photon resonance near $400 \mathrm{~nm}$, while the gold plasmon resonance peak is near $530 \mathrm{~nm}$. The SHG signal from the molecular dye dissolved in water is due to incoherent hyper-Rayleigh scattering (HRS), where the SHG signal is proportional to the molecular concentration. ${ }^{52-58}$ Enhanced SHG from the nanoGUMBOS as compared with the molecular dye is attributed primarily to the increased size. To a first approximation, the SHG signal is expected to scale as the radius to the sixth power for small nanoparticles, ${ }^{51,59}$ although many other factors are important including the molecular nonlinear susceptibilities and angular scattering distributions. While SHG is a coherent process for a single nanoparticle, the measured SHG signal from a colloidal nanoparticle sample is an incoherent sum of the nanoparticles in the laser focus such that the total SHG signal is linearly proportional to the nanoparticle concentration..$^{50,51,56-58}$ The nanoGUMBOS also have a higher extinction at $800 \mathrm{~nm}$ as compared with the BG molecule and the gold nanospheres, causing some single-photon resonance enhancement in the nonlinear spectroscopy. Local ordering of the BG molecules in the nanoGUMBOS due to aggregation and $\pi-\pi$ stacking may also occur causing orientations that are not isotropically distributed, leading to an additional source of increased SHG signals. Additional measurements are needed to quantify the SHG signal per nanoparticle for the nanoGUMBOS sample, although estimates based on the nanoparticle material densities indicate that the gold nanoparticles have a higher SHG signal per nanoparticle. Unlike the gold nanospheres and the BG dye in water, a THG signal can be clearly observed from the colloidal nanoGUMBOS sample. The enhanced THG is attributed to an increased $\chi^{(3)}$ as well as the enhanced SHG coherently adding with the fundamental through sum-frequency generation with $\omega+2 \omega$ $=3 \omega$, although more work is needed to better understand the details of these enhanced nonlinear optical processes. ${ }^{23,60}$ Additional research is also needed to investigate the sizedependence of nonlinear optical signals from nanoGUMBOS for designing and optimizing potential nonlinear optical probes.

\section{CONCLUSION}

Synthesis, characterization, excited-state dynamics, and nonlinear spectroscopy of $30 \mathrm{~nm}$ colloidal [BG][BETI] nanoGUMBOS in water are studied and reported. The [BG][BETI] nanoGUMBOS sample exhibits enhanced fluorescence emission in the NIR region. This enhanced fluorescence emission, longer $S_{1}$ excited-state lifetimes, and a red-shifted $S_{1}$ to $S_{0}$ transition of the brilliant green molecule in the nanoGUMBOS medium as compared with the molecular dye in water is attributed to molecular hindrance, aggregation, and an altered chemical environment. The [BG][BETI] nanoGUMBOS also show enhanced second harmonic generation and third harmonic generation signals as compared with the molecular dye and colloidal gold nanospheres in water due to very large effective nonlinear susceptibilities. The results from the study of these novel molecular-based nanomaterials demonstrate that $[\mathrm{BG}][\mathrm{BETI}]$ nanoGUMBOS are excellent candidates for sensing, imaging, and labeling applications in biological systems.

\section{SUPPLEMENTARY MATERIAL}

See supplementary material for additional details on the characterization of the GUMBOS, the nanoGUMBOS, and the gold nanospheres, as well as on the instrumentation.

\section{ACKNOWLEDGMENTS}

Generous financial support for this work was provided by Louisiana State University. L.H.H. acknowledges financial support from the National Science Foundation EPSCoR CIMM project under Award No. OIA-1541079. I.M.W. acknowledges financial support from the National Science Foundation under Grant Nos. CHE-1307611 and DMR0843962.

${ }^{1}$ I. M. Warner, B. El-Zahab, and N. Siraj, Anal. Chem. 86(15), 7184-7191 (2014).

${ }^{2}$ P. E. Kolic, N. Siraj, S. Hamdan, B. P. Regmi, and I. M. Warner, J. Phys. Chem. C 120(9), 5155-5163 (2016).

${ }^{3}$ T. E. Karam, N. Siraj, I. M. Warner, and L. H. Haber, J. Phys. Chem. C 119(50), 28206-28213 (2015).

${ }^{4}$ D. K. Bwambok, B. El-Zahab, S. K. Challa, M. Li, L. Chandler, G. A. Baker, and I. M. Warner, ACS Nano 3(12), 3854-3860 (2009).

${ }^{5}$ C. Lu, S. Das, P. K. Magut, M. Li, B. El-Zahab, and I. M. Warner, Langmuir 28(40), 14415-14423 (2012).

${ }^{6}$ B. P. Regmi, J. Monk, B. El-Zahab, S. Das, F. R. Hung, D. J. Hayes, and I. M. Warner, J. Mater. Chem. 22(27), 13732-13741 (2012).

${ }^{7}$ B. P. Regmi, W. I. S. Galpothdeniya, N. Siraj, M. H. Webb, N. C. Speller, and I. M. Warner, Sens. Actuators, B 209, 172-179 (2015).

${ }^{8}$ C. Szent-Gyorgyi, B. F. Schmidt, Y. Creeger, G. W. Fisher, K. L. Zakel, S. Adler, J. A. Fitzpatrick, C. A. Woolford, Q. Yan, and K. V. Vasilev, Nat. Biotechnol. 26(2), 235-240 (2008).

${ }^{9}$ J. R. Babendure, S. R. Adams, and R. Y. Tsien, J. Am. Chem. Soc. 125(48), 14716-14717 (2003).

${ }^{10}$ A. Punzi, G. Martin-Gassin, J. Grilj, and E. Vauthey, J. Phys. Chem. C 113(27), 11822-11829 (2009).

${ }^{11}$ A. C. Bhasikuttan, A. V. Sapre, and T. Okada, J. Phys. Chem. A 107(17), 3030-3035 (2003).

${ }^{12}$ P. Fita, A. Punzi, and E. Vauthey, J. Phys. Chem. C 113(48), 20705-20712 (2009).

${ }^{13}$ D. M. Kolpashchikov, J. Am. Chem. Soc. 127(36), 12442-12443 (2005).

${ }^{14}$ J. A. Fitzpatrick, Q. Yan, J. J. Sieber, M. Dyba, U. Schwarz, C. Szent-Gyorgyi, C. A. Woolford, P. B. Berget, A. S. Waggoner, and M. P. Bruchez, Bioconjugate Chem. 20(10), 1843-1847 (2009).

${ }^{15}$ J. S. Paige, K. Y. Wu, and S. R. Jaffrey, Science 333(6042), 642-646 (2011).

${ }^{16}$ A. C. Bhasikuttan, J. Mohanty, W. M. Nau, and H. Pal, Angew. Chem. 119(22), 4198-4200 (2007).

${ }^{17}$ W. Xu and Y. Lu, Anal. Chem. 82(2), 574-578 (2009).

${ }^{18}$ T. Funada, T. Hirose, N. Tamai, and H. Yao, Phys. Chem. Chem. Phys. 17(16), 11006-11013 (2015).

${ }^{19}$ V. Berger, Phys. Rev. Lett. 81(19), 4136 (1998).

${ }^{20}$ R. Boyd, Nonlinear Optics (Academic Press, New York, 1992).

${ }^{21}$ Y.-R. Shen, The Principles of Nonlinear Optics (Wiley-Interscience, New York, 1984), 575, p. 1.

${ }^{22}$ N. Broderick, G. Ross, H. Offerhaus, D. Richardson, and D. Hanna, Phys. Rev. Lett. 84(19), 4345 (2000).

${ }^{23}$ B. Corcoran, C. Monat, C. Grillet, D. J. Moss, B. J. Eggleton, T. White, L. O'Faolain, and T. F. Krauss, Nat. Photonics 3(4), 206-210 (2009).

${ }^{24}$ S. I. Stupp, V. LeBonheur, K. Walker, L.-S. Li, K. E. Huggins, M. Keser, and A. Amstutz, Science 276(5311), 384-389 (1997).

${ }^{25}$ P. J. Campagnola and L. M. Loew, Nat. Biotechnol. 21(11), 1356-1360 (2003)

${ }^{26}$ Y. Pu, R. Grange, C.-L. Hsieh, and D. Psaltis, Phys. Rev. Lett. 104(20), 207402 (2010). 
${ }^{27}$ X. Chen, O. Nadiarynkh, S. Plotnikov, and P. J. Campagnola, Nat. Protocols 7(4), 654-669 (2012).

${ }^{28}$ P. Pantazis, J. Maloney, D. Wu, and S. E. Fraser, Proc. Natl. Acad. Sci. U. S. A. 107(33), 14535-14540 (2010).

${ }^{29}$ R. D. Wampler, D. J. Kissick, C. J. Dehen, E. J. Gualtieri, J. L. Grey, H.-F. Wang, D. H. Thompson, J.-X. Cheng, and G. J. Simpson, J. Am. Chem. Soc. 130(43), 14076-14077 (2008).

${ }^{30}$ J. E. Reeve, H. A. Collins, K. D. Mey, M. M. Kohl, K. J. Thorley, O. Paulsen, K. Clays, and H. L. Anderson, J. Am. Chem. Soc. 131(8), 2758-2759 (2009).

${ }^{31}$ N. Siraj, B. El-Zahab, S. Hamdan, T. E. Karam, L. H. Haber, M. Li, S. O. Fakayode, S. Das, B. Valle, and R. M. Strongin, Anal. Chem. 88(1), 170-202 (2015).

${ }^{32}$ L. H. Haber, S. J. Kwok, M. Semeraro, and K. B. Eisenthal, Chem. Phys. Lett. 507(1), 11-14 (2011).

${ }^{33}$ L. H. Haber and K. B. Eisenthal, J. Phys. Chem. B 117(16), 4249-4253 (2012).

${ }^{34}$ G. Gonella and H.-L. Dai, Phys. Rev. B 84(12), 121402 (2011).

${ }^{35}$ S.-H. Jen and H.-L. Dai, J. Phys. Chem. B 110(46), 23000-23003 (2006).

${ }^{36}$ E. Génin, Z. Gao, J. A. Varela, J. Daniel, T. Bsaibess, I. Gosse, L. Groc, L. Cognet, and M. Blanchard-Desce, Adv. Mater. 26(14), 2258-2261 (2014).

${ }^{37}$ K. Amro, J. Daniel, G. Clermont, T. Bsaibess, M. Pucheault, E. Genin, M. Vaultier, and M. Blanchard-Desce, Tetrahedron 70(10), 1903-1909 (2014).

${ }^{38}$ J. Daniel, F. Bondu, F. d. r. Adamietz, M. Blanchard-Desce, and V. Rodriguez, ACS Photonics 2(8), 1209-1216 (2015).

${ }^{39}$ R. R. Kumal, T. E. Karam, and L. H. Haber, J. Phys. Chem. C 119(28), 16200-16207 (2015).

${ }^{40}$ T. E. Karam and L. H. Haber, J. Phys. Chem. C 118, 642-649 (2013).

${ }^{41}$ T. E. Karam, H. T. Smith, and L. H. Haber, J. Phys. Chem. C 119, 18573-18580 (2015).
${ }^{42}$ T. E. Karam, R. A. Khoury, and L. H. Haber, J. Chem. Phys. 144(12), 124704 (2016).

${ }^{43}$ M. Yoshizawa, K. Suzuki, A. Kubo, and S. Saikan, Chem. Phys. Lett. 290(1), 43-48 (1998).

${ }^{44}$ Y. Nagasawa, Y. Ando, and T. Okada, Chem. Phys. Lett. 312(2), 161-168 (1999).

${ }^{45}$ Y. Nagasawa, Y. Ando, D. Kataoka, H. Matsuda, H. Miyasaka, and T. Okada, J. Phys. Chem. A 106(10), 2024-2035 (2002).

${ }^{46}$ D. Bingemann and N. P. Ernsting, J. Chem. Phys. 102(7), 2691-2700 (1995).

${ }^{47}$ R. R. Kumal, C. R. Landry, M. Abu-Laban, D. J. Hayes, and L. H. Haber, Langmuir 31(36), 9983-9990 (2015).

${ }^{48}$ J. Nappa, I. Russier-Antoine, E. Benichou, C. Jonin, and P. Brevet, J. Chem. Phys. 125(18), 184712 (2006).

${ }^{49}$ F. W. Vance, B. I. Lemon, and J. T. Hupp, J. Phys. Chem. B 102(50), 10091-10093 (1998).

${ }^{50}$ K. B. Eisenthal, Chem. Rev. 106(4), 1462-1477 (2006).

${ }^{51}$ J. I. Dadap, J. Shan, K. B. Eisenthal, and T. F. Heinz, Phys. Rev. Lett. 83(20), 4045 (1999).

${ }^{52}$ Y. Rao, X. M. Guo, Y. S. Tao, and H. -F. Wang, J. Phys. Chem. A 108(39), 7977-7982 (2004).

${ }^{53}$ H. Wang, E. C. Y. Yan, E. Borguet, and K. B. Eisenthal, Chem. Phys. Lett. 259(1-2), 15-20 (1996).

${ }^{54}$ R. Macovez, N. Lopez, M. Mariano, M. Maymò, and J. Martorell, J. Phys. Chem. C 116(51), 26784-26790 (2012).

${ }^{55}$ P. Kaatz and D. P. Shelton, J. Chem. Phys. 105(10), 3918-3929 (1996).

${ }^{56}$ S. Roke and G. Gonella, Annu. Rev. Phys. Chem. 63, 353-378 (2012).

${ }^{57}$ S.-H. Jen, G. Gonella, and H.-L. Dai, J. Phys. Chem. A 113, 4758-4762 (2009).

${ }^{58}$ R. R. Kumal, H. Nguyenhuu, J. E. Winter, R. L. McCarley, and L. H. Haber, J. Phys. Chem. C 121, 15851-15860 (2017).

${ }^{59}$ A. G. F. de Beer and S. Roke, Phys. Rev. B 79, 155420 (2009).

${ }^{60}$ S.-N. Zhu, Y.-Y. Zhu, and N.-B. Ming, Science 278(5339), 843-846 (1997). 